\title{
Confirmation that Rickettsia helvetica sp. nov. Is a Distinct Species of the Spotted Fever Group of Rickettsiae
}

\author{
LORENZA BEATI, ${ }^{1}$ OLIVIER PÉTER,${ }^{2}$ WILLY BURGDORFER, ${ }^{3}$ ANDRÉ AESCHLIMANN, ${ }^{4}$ \\ AND DIDIER RAOULT ${ }^{1 *}$ \\ Unité des Rickettsies, Faculté de Médecine, 27, Boulevard Jean Moulin, 13385 Marseille, France ${ }^{1}$; \\ Institut Central des Hôpitaux Valaisans, Microbiologie Clinique, 1950 Sion, ${ }^{2}$ and Institut de Zoologie, \\ Université de Neuchâtel, 2000 Neuchâtel, ${ }^{4}$ Switzerland; and Rocky Mountain Laboratories, \\ National Institutes of Health, Hamilton, Montana $59840^{3}$
}

\begin{abstract}
We propose the name Rickettsia helvetica sp. nov. for a rickettsial serotype of unknown pathogenicity isolated in 1979 in Switzerland from Ixodes ricinus ticks and designated the Swiss agent. The growth characteristics and the results of microimmunofluorescence serologic typing, sodium dodecyl sulfate-polyacrylamide gel electrophoresis, Western blotting (immunoblotting) with specific mice sera, and a polymerase chain reaction followed by restriction fragment length polymorphism analysis confirmed previously reported preliminary findings which suggested that this rickettsia, to which a name was given provisionally, does represent a new member of the spotted fever group of rickettsiae. The type strain is C3 (Reference Center for Rickettsioses, Marseille, France).
\end{abstract}

In 1979 a strain of rickettsia isolated from Lxodes ricinus in Switzerland was identified as a previously undescribed organism belonging to the spotted fever group (SFG) and was designated the Swiss agent (4). Isolation was accomplished by intraperitoneal inoculation of newly molted nymphal ticks that were presumably transovarially infected into meadow voles (Microtus pennsylvanicus). The tunicae vaginalis and spleens of the infected meadow voles were then inoculated into yolk sacs of embryonated hen eggs. Identification based on indirect microimmunofluorescence (MIF) serologic typing (16) and microagglutination (5) data was accomplished by comparing the isolate with prototype strains belonging to the following rickettsial species: Rickettsia conorii, Rickettsia rickettsii, Rickettsia sibirica, Rickettsia slovaca, Rickettsia montana, Rickettsia australis, Rickettsia akari, Rickettsia rhipicephali, Rickettsia parkeri, Rickettsia typhi, Rickettsia prowazekii, and Rickettsia canada. No cross-reactions with any of the other SFG rickettsiae were observed by in MIF tests performed with serum raised in mice against the Swiss agent, and none of the sera specific for the other SFG rickettsiae reacted with the Swiss agent. As determined by the Gimenez staining method (6), this isolate was described as a diplococcal or rod-shaped intracellular organism, and it could not be differentiated from other rickettsial species by electron microscopy $(4,8)$. The Swiss agent grew well in yolk sacs, chicken embryo fibroblasts, and Vero cells without producing cytopathic effects. It exhibited moderate pathogenicity when it was inoculated into meadow voles, but rabbits, guinea pigs, and Swiss mice did not seem to be susceptible.

In a separate study (15), Western blot (immunoblot) anal$y$ sis of the protein profiles of rabbit antisera was used to compare this rickettsia with $R$. rickettsii, $R$. sibirica, $R$. slovaca, and $R$. conorii. The Swiss agent proved to be a new rickettsia, and the name "Rickettsia helvetica" was tentatively proposed but never validated.

In this study we reexamined this rickettsia (strain $\mathrm{C}^{\mathrm{T}}[\mathrm{T}$ $=$ type strain]). We studied its intracellular behavior by

${ }^{*}$ Corresponding author. using double fluorescence labelling of $\mathrm{F}$ actin and organisms (19). Strain $C 3^{\mathbf{T}}$ was compared with two new isolates obtained from $I$. ricinus collected in Switzerland (strains Ix 10 and Ix 12). The sodium dodecyl sulfate (SDS)-polyacrylamide gel electrophoresis (PAGE) profiles of these organisms were compared with the profiles of all of the available SFG rickettsiae. MIF serologic typing was repeated by using all of the reference rickettsial strains which were not used in 1979. Mouse serum against the Swiss agent, which is more species specific (16) than rabbit sera (1), was used for Western blot analysis. A polymerase chain reaction and restriction fragment length polymorphism analysis of the DNA of this rickettsia was also performed (17). Our results confirm the previous description of the Swiss agent as a new SFG tick-borne rickettsia, for which we officially propose the name Rickettsia helvetica.

\section{MATERIALS AND METHODS}

Rickettsial strains. $R$. conorii Moroccan strain VR-141, $R$. rickettsii Sheila Smith VR-149, and $R$. akari VR-148 were obtained from the American Type Culture Collection. $R$. sibirica $232, R$. australis, $R$. slovaca, $R$. rhipicephali, the Israeli spotted fever rickettsia (7), and the Thai tick typhus rickettsia (18) were supplied by G. A. Dasch, Naval Medical Research Institute, Bethesda, Md. R. montana, Rickettsia bellii, and Rickettsia japonica were obtained from D. H. Walker, University of Texas, Galveston. $R$. helvetica type strain C3 was isolated from $I$. ricinus ticks collected in the Staatswald, Bern, Switzerland. Strains Ix 10 and Ix 12 were isolated by the shell vial assay $(10,14)$ from two $I$. ricinus ticks collected in two different regions (Ticino and Neuchâtel, Switzerland). The recently described organisms "Rickettsia massiliae", (proposed name) isolate Mtu5 $(2,3)$ and "Rickettsia africae" (proposed name) (9) were also included in the study. In each case, heavily infected L929 or Vero cells from $10150-\mathrm{cm}^{2}$ tissue culture flasks were harvested and stored at $-80^{\circ} \mathrm{C}$ until they were used for polymerase chain reaction amplification, SDS-PAGE, Western blotting, and mouse immunization. The strains were cultivated in 
Earle's minimum essential medium containing $4 \%$ fetal calf serum and $2 \mathrm{mM}$ L-glutamine at $32^{\circ} \mathrm{C}$.

Double fluorescence labelling of $\mathrm{F}$ actin and organisms. For double fluorescence labelling of F actin and organisms (19), confluent monolayers of Vero cells grown in shell vials were infected with $R$. helvetica. At $48 \mathrm{~h}$ after infection, the cells were washed three times (10 min each time) in phosphatebuffered saline ( $\mathrm{pH} 7.4$ ) (PBS), fixed with 3\% paraformaldehyde for $20 \mathrm{~min}$, and washed again in PBS. The cells were permeabilized with $0.1 \%$ Triton X-100 for $1 \mathrm{~min}$ and washed with PBS. Polyclonal mouse serum to $R$. helvetica (diluted 1/50) and fluorescein isothiocyanate-labelled goat antimouse conjugate (diluted $1 / 100$ in PBS; Immunotec, Marseille, France) were used to label the bacteria. $F$ actin was labelled with a solution $(10 \mathrm{U} / \mathrm{ml})$ of $N$-(7-nitrobenz2-oxa-1,3-diazol-4-yl)-phallacidin (Interchim, Montluçon, France) in PBS. The slides with the cell monolayers were then observed by fluorescence microscopy at a magnification of $\times 400$ in order to detect actin polymerization, which provided evidence of intracellular movements.

Mouse immunization. Using the method described previously (16), we produced polyclonal antisera by inoculating groups of five Swiss mice intravenously with $R$. bellii, " $R$. massiliae," " $R$. africae," $R$. japonica, the Israeli spotted fever rickettsia, the Thai tick typhus rickettsia, isolate Mtu5, and the Swiss agent $\left(0.1 \mathrm{ml}\right.$ containing about $10^{3}$ to $10^{4}$ organisms). The mice were boosted on day 7 with a similar inoculum. On day 10 , the mice were anesthetized and exsanguinated by cardiac puncture. The sera from each group of five mice were pooled and stored at $-20^{\circ} \mathrm{C}$.

MIF serotyping. The MIF test was performed and the specificity difference (SPD) was calculated by using the method of Philip et al. (16). $R$. helvetica was compared with $R$. bellii, " $R$. africae," $R$. massiliae, the Israeli spotted fever rickettsia, isolate Mtu5, the Thai tick typhus rickettsia, and $R$. japonica. Briefly, the polyclonal mouse antisera (twofold dilutions) were incubated with both homologous and heterologous antigens (infected cells). Binding of sera was detected by using fluorescein isothiocyanate-labelled goat antimouse immunoglobulins $\mathrm{G}$ and $\mathrm{M}$ (Immunotec) diluted 1/100 in PBS. The highest serum dilutions giving positive reactions were recorded as endpoint titers: $\mathrm{SPD}=(\mathrm{Aa}+\mathrm{Bb})-(\mathrm{Ab}+$ $\mathrm{Ba}$ ), where $\mathrm{Aa}$ or $\mathrm{Bb}$ is $-\log _{2}$ of the endpoint titer between serum $\mathrm{A}$ or $\mathrm{B}$ and homologous antigen $\mathrm{a}$ or $\mathrm{b}$ and $\mathrm{Ab}$ or $\mathrm{Ba}$ is $-\log _{2}$ of the endpoint titer of serum $A$ or $B$ against heterologous antigen $b$ or $a$. If the SPD was less than 3 , the two strains were assumed to be identical; if the SPD was 3 or greater, the strains were different.

SDS-PAGE and Western blot immunoassay. The contents of 10 flasks infected with $R$. conorii, $R$. sibirica, $R$. slovaca, $R$. rhipicephali, $R$. akari, $R$. australis, $R$. montana, $R$. massiliae, $R$. helvetica $\mathrm{C}^{\mathrm{T}}$, strain $\mathrm{Ix} 10$, and strain $\mathrm{Ix} 12$ were sonicated and centrifuged at $150 \times g ; 20 \mathrm{ml}$ of each supernatant was layered onto $20 \mathrm{ml}$ of $25 \%$ sucrose in PBS, and the preparation was centrifuged at $7,000 \times g$ for $30 \mathrm{~min}$. The pellet was purified on a Renografin density gradient (22), and the rickettsiae collected were washed three times in PBS 10 min each time (by centrifugation at $17,500 \times g$ ) and resuspended in distilled water. The protein concentration was adjusted to $1 \mathrm{mg} / \mathrm{ml}$ (Total Protein Test; Bio-Rad, Richmond, Calif.). For SDS-PAGE, $5 \mu$ l of each purified antigen was dissolved in $5 \mu$ l of Laemmli (11) solution (4\% SDS, $10 \%$ 2-mercaptoethanol, $0.5 \%$ bromo-phenol blue, $0.125 \mathrm{M}$ Tris hydrochloride [pH 6.8], 25\% glycerol) at room temperature. SDS-PAGE was performed with an $8 \%$ separating gel and a $3.9 \%$ stacking gel. The gel was electrophoresed in a Mini-
Protean II cell (Bio-Rad) at $10 \mathrm{~mA}$ in an ice bath, and the protein bands were visualized with Coomassie brilliant blue stain. High-molecular-weight standards (Bio-Rad) were used to estimate the molecular weights of the protein bands. The results were compared with the previously obtained profiles of the strains that we did not use in this study by using a computer system (The Imager; Appligene, Illkich, France) and the QGEL-DTM program (Quantigel Corp., Madison, Wis.), which allowed us to calculate the molecular weights and to compare the new profiles with all of the profiles recorded previously (3). An identical gel was transferred to nitrocellulose paper (20) in a Trans-blot apparatus (Bio-Rad) by electrophoresis at $50 \mathrm{~V}$ for $1 \mathrm{~h}$ in an ice bath. Nonspecific binding sites were blocked by incubating the paper overnight in 5\% nonfat dry milk in TBS (10 mM Tris hydrochloride [pH 7.5], $250 \mathrm{mM} \mathrm{NaCl}, 0.01 \%$ Merthiolate). After three 10-min washes in TBS, the nitrocellulose paper was overlaid with mouse serum against the Swiss agent diluted $1 / 100$ in $3 \%$ nonfat dry milk in TBS, and the preparation was left on a rocker for $2 \mathrm{~h}$ at room temperature. Unfixed antibodies were removed by three $10-\mathrm{min}$ washes in TBS. The paper was then left on a rocker for $2 \mathrm{~h}$ in $3 \%$ milk in TBS containing goat anti-mouse globulin peroxidase conjugate (Immunotec) diluted 1/100. After three additional 10-min washes in TBS, the bound peroxidase was detected by using a solution containing $0.015 \%$ 4-chloro-1-naphthol, $0.015 \%$ hydrogen peroxide, and $16 \%$ methanol in TBS. As soon as the bands were visible, the reaction was stopped with distilled water.

Polymerase chain reaction amplification, DNA digestion, and electrophoresis. For polymerase chain reaction amplification, DNA digestion, and electrophoresis (17), 1-ml portions of Vero cells infected with $R$. helvetica, Ix10, and Ix12 were centrifuged three times in distilled water at $17,500 \times \mathrm{g}$ for $5 \mathrm{~min}$. Each final pellet was resuspended in distilled water and boiled for $10 \mathrm{~min}$ according to a simplified sample protocol (21). A sample containing $10 \mu \mathrm{l}$ of the boiled suspension, $59.5 \mu \mathrm{l}$ of distilled water, $10 \mu \mathrm{l}$ of Taq buffer (Boehringer-Mannheim, Meylan, France), $10 \mu \mathrm{l}$ of deoxynucleotide triphosphates ( $2 \%$ dATP, $2 \%$ dCTP, $2 \%$ dGTP, and $2 \%$ dTTP in distilled water; Boehringer-Mannheim), $5 \mu \mathrm{l}$ of each component of the primer pair (Rp. CS.877p-1258n and $R r$. $190.70 \mathrm{p}-602 \mathrm{n})$, and $0.5 \mu \mathrm{l}$ of $T a q$ polymerase $(5,000$ $\mathrm{U} / \mathrm{ml}$; Boehringer-Mannheim) was subjected to 15 cycles of denaturation $\left(95^{\circ} \mathrm{C}\right.$ for $\left.20 \mathrm{~s}\right)$, annealing $\left(48^{\circ} \mathrm{C}\right.$ for $\left.30 \mathrm{~s}\right)$, and sequence extension $\left(60^{\circ} \mathrm{C}\right.$ for $\left.2 \mathrm{~min}\right)$ in a thermal cycler (PREM III Lep Scientific, Flobio, Courbevoie, France), replenished with $0.5 \mu$ l of Taq polymerase, and subjected to an additional 15 cycles. The results of amplification were visualized on a $1.5 \%$ agarose gel after electrophoretic migration ( $100 \mathrm{~V}$ for $1 \mathrm{~h}$ ) of $10 \mu \mathrm{l}$ of the amplified material. The agarose gels were stained with ethidium bromide and examined by using UV transillumination. Then, $23.3 \mu \mathrm{l}$ of the amplification products was incubated for $2 \mathrm{~h}$ at $37^{\circ} \mathrm{C}$ with 1 $\mu l$ of restriction endonucleases; the digested products were separated by electrophoresis on $8 \%$ polyacrylamide gels at $80 \mathrm{~V}$ for 4 to $5 \mathrm{~h}$ in a Bio-Rad apparatus, stained with ethidium bromide, and examined by using UV transillumination. DNA molecular weight marker V (BoehringerMannheim) was used to estimate the sizes of the observed DNA fragments. The polymerase chain reaction-restriction fragment length polymorphism analysis was performed by using the oligonucleotide primer pair (Bioprobe Systems, Montreuil-sous-bois, France) and restriction endonuclease (New England Biolabs, Beverly, Mass.) proposed by Regnery et al. (17) for the differentiation of SFG rickettsiae: $R \boldsymbol{p}$. CS.877p-1258n (coding for a 381-bp sequence) and AluI. The 
TABLE 1. MIF antibody titers and SPDs obtained from reciprocal cross-reactions of mouse antisera with SFG rickettsiae

\begin{tabular}{|c|c|c|c|c|c|c|c|c|}
\hline \multirow[b]{2}{*}{ Antigen } & \multicolumn{8}{|c|}{ MIF antibody titer (SPD) with ${ }^{a}$ : } \\
\hline & 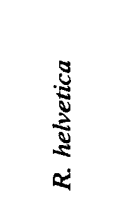 & 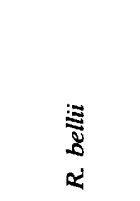 & 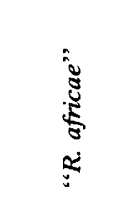 & 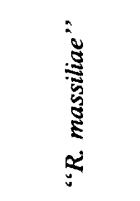 & 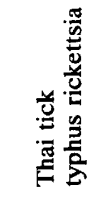 & 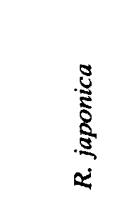 & 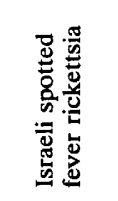 & 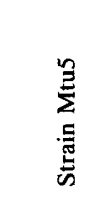 \\
\hline$R$. helvetica & $1,024(0)$ & $256(8)$ & $64(7)$ & $64(9)$ & $128(4)$ & $256(5)$ & $128(5)$ & $128(5)$ \\
\hline R. bellii & $32(8)$ & $2,048(0)$ & $<8(11)$ & $<8(12)$ & $<8(9)$ & $<8(11)$ & $<8(10)$ & $<8(10)$ \\
\hline "R. africae" & $128(7)$ & $256(11)$ & $1,024(0)$ & 64 (13) & $32(6)$ & $128(6)$ & $128(5)$ & $64(6)$ \\
\hline "R. massiliae" & $64(8)$ & $256(6)$ & $<8(13)$ & $2,048(0)$ & $<8(10)$ & $64(7)$ & $32(8)$ & $64(7)$ \\
\hline Thai tick typhus rickettsia & $128(4)$ & $256(9)$ & $128(6)$ & $128(10)$ & $256(0)$ & $256(4)$ & $64(4)$ & $64(4)$ \\
\hline R. japonica & $12(5)$ & 256 (11) & $128(6)$ & $256(7)$ & $64(4)$ & $1,024(0)$ & $64(5)$ & $64(5)$ \\
\hline Israeli spotted fever rickettsia & $128(5)$ & $256(10)$ & $128(5)$ & $128(8)$ & $128(4)$ & $256(5)$ & $512(0)$ & $32(7)$ \\
\hline Strain Mtu5 & $128(5)$ & $256(10)$ & $64(6)$ & $128(7)$ & $128(4)$ & $256(5)$ & $64(7)$ & $512(0)$ \\
\hline
\end{tabular}

${ }^{a}$ The titers are the reciprocals of the highest dilutions of antisera that gave positive reactions. The SPDs were calculated by using the formula given in the text.

results were compared with previously reported data and with the results in our data bank, which contains the profiles of the reference strains obtained with The Imager system (Appligene) and the QGEL-DTM program (Quantigel Corp.) $(3,17)$.

\section{RESULTS AND DISCUSSION}

$R$. helvetica and isolates $\mathrm{Ix} 10$ and $\mathrm{Ix} 12$ from $I$. ricinus do not produce cytopathic changes in Vero cells, and they can be continuously subcultured by trypsinization of the monolayers. The growth conditions are identical to those of other SFG rickettsiae (i.e., Earle's minimum essential medium supplemented with $4 \%$ fetal calf serum and $2 \mathrm{mM}$ L-glutamine). $R$. helvetica can be stored at $-80^{\circ} \mathrm{C}$ without losing its viability. It grows well in Vero and L929 cells. However, $R$. helvetica and isolates IX 10 and IX 12 have a characteristic not observed previously for other rickettsiae belonging to the SFG: they disappear from the human embryonic lung fibroblast monolayers after 1 to 2 days of culture. This observation provides a way to differentiate $R$. helvetica from all other SFG rickettsiae. Thus, it was necessary to grow L929 cells in shell vials before we could successfully isolate rickettsiae from $I$. ricinus ticks. In the classical shell vial technique (14), isolation attempts are usually performed with human embryonic lung fibroblast cells, whose slow growth potentiates the growth of rickettsiae; L929 cells grow more quickly so that frequent medium changes (every 4 to 5 days) are necessary to avoid disruption of the monolayers $(3,10$, 14).

After double labelling $R$. helvetica and the actin filaments (19), we did not observe any actin tail connected to the organisms, so we have no evidence of actin polymerization. This could mean that $R$. helvetica cannot move inside cells and therefore it is unlikely that it can invade nuclei (19).

MIF test. The results of the MIF test (reciprocal titers and SPDs) are shown in Table 1. The SPDs between $R$. helvetica and the other rickettsiae were always more than 3 . We did not infect mice with isolates Ix 10 and Ix 12, but antisera against $R$. helvetica recognized both of these strains at the same titer as they recognized $R$. helvetica $(1 / 1,024)$, while the heterologous titers were always lower $(1 / 32$ to $1 / 128)$. These results show that $R$. helvetica and isolates Ix 10 and $\mathbf{~ X}$ 12 are identical. All of the rickettsiae included in our MIF assay, as well as the rickettsiae studied in the previous report (4), can be considered members of different serotypes
$(4 \leq$ SPDs $\leq 13)$ and, on the basis of the usual taxonomic criteria (16), represent different species.

SDS-PAGE. The proteins of $R$. helvetica and isolates Ix 10 and Ix 12 produced identical electrophoretic mobility patterns that are different from those of all other rickettsiae. The differences are evident in the high-molecular-weight range. The protein bands corresponded to the following molecular masses (the most prominent bands are underlined): 115 and $130 \mathrm{kDa}$ for $R$. conorii; $112,114,135,140$, and $145 \mathrm{kDa}$ for $R$. sibirica; $107,115,125,135$, and $140 \mathrm{kDa}$ for $R$. slovaca; 110 , and $140 \mathrm{kDa}$ for $R$. australis; $90,112,135$, and $145 \mathrm{kDa}$ for $R$. akari; $100,110,140$, and $155 \mathrm{kDa}$ for $R$. rhipicephali; 110,130 , and $150 \mathrm{kDa}$ for " $R$. massiliae"; 113 , $115, \underline{135}, 145$, and $155 \mathrm{kDa}$ for $R$. japonica; 135 and $140 \mathrm{kDa}$ for $R$. montana; and $95,100,110,130,145$, and $150 \mathrm{kDa}$ for $R$. helvetica. Compared with the profiles of the rickettsiae studied in a previous report (3) and with the data collected in our data bank, the profile of $R$. helvetica appears to be unique (Fig. 1).

Western blotting. Western blotting was performed with a mouse antiserum against $R$. helvetica that had a homologous titer of $1 / 1,024$ (Fig. 2). The reaction to the homologous antigen was clearly more intense than the reactions observed with heterologous serotypes. With the high-molecularweight species-specific protein bands, the serum reacted to two major bands of the $R$. helvetica and isolate $\mathrm{Ix} 10$ and $\mathrm{Ix}$ 12 antigens (molecular masses of 100 [prominent band], 120, and sometimes $140 \mathrm{kDa}$ ). In the high-molecular-weight range, the only heterologous cross-reaction was observed with $R$. japonica (molecular mass, $115 \mathrm{kDa}$ [prominent band]), while in the lower-molecular-weight range $(<50$ $\mathrm{kDa}$ ), weak cross-reactions were observed between this serum and the antigens of almost all other rickettsiae. The SDS-PAGE and Western blotting profiles confirm that the polypeptides of $R$. helvetica have characteristics similar to those of the polypeptides of the SFG rickettsiae mainly in the low-molecular-weight range. This seems to confirm the low heterologous cross-reactive titers obtained with the $R$. helvetica serum (Table 1). These results support the hypothesis that $R$. helvetica is a new rickettsia and do not confirm the previously advanced hypothesis that the Swiss agent is closely related to the European SFG rickettsiae (15). This discrepancy has to be ascribed to the fact that the latter hypothesis was based on Western blotting results obtained with polyclonal rabbit sera, which usually contain more 


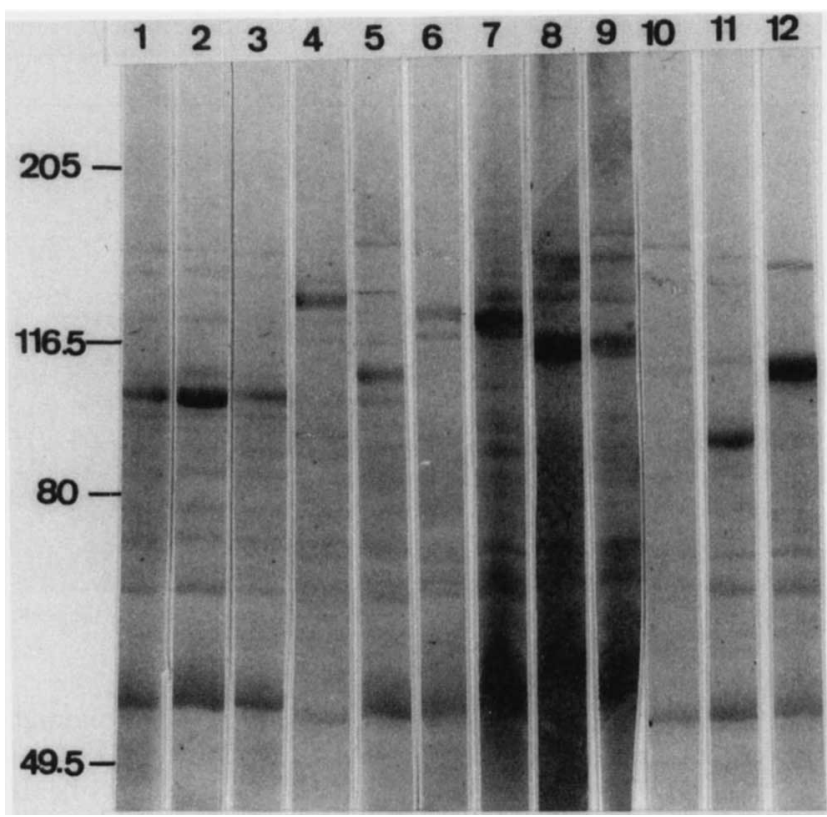

FIG. 1. Coomassie brilliant blue-stained SDS-PAGE profiles of $R$. helvetica (Swiss agent) strain $\mathrm{C}^{\mathrm{T}}$ (lane 1), tick isolate Ix 10 (lane 2 ), tick isolate Ix 12 (lane 3), $R$. montana (lane 4), $R$. rhipicephali (lane 5), $R$. conorii (lane 6), $R$. slovaca (lane 7), $R$. sibirica (lane 8 ), $R$. japonica (lane 9), " $R$. massiliae" (lane 10), $R$. akari (lane 11), and $R$. australis (lane 12). Molecular masses (in kilodaltons) are indicated on the left.

antibodies that cross-react with lipopolysaccharide-like antigens than mouse sera, which are more reactive with species-specific epitopes $(1,16,23)$.

Polymerase chain reaction-restriction fragment length poly- morphism analysis. The DNAs of $R$. helvetica and isolates Ix 10 and Ix 12 could be amplified only with the citrate synthase primers (Rp. CS.877p-1258n). Digestion by AluI of these amplified materials (Fig. 3) gave profiles identical to those observed for the SFG rickettsiae (17). The $R r$. 190.70p-602n primer pair was unable to amplify the DNAs of these rickettsial strains, as described previously for other rickettsiae, including $R$. akari, $R$. australis, and $R$. bellii (17). According to the criteria proposed by Regnery et al. (17), $R$. helvetica can be considered an SFG rickettsia because the restriction profiles obtained after citrate synthase amplification and $A l u I$ digestion are identical to those of all other SFG rickettsiae. However, the gene coding for the $190-\mathrm{kDa}$ protein is unable to amplify the DNA of $R$. helvetica, suggesting that the major differences are located in the outer membrane high-molecular-weight surface proteins.

Justification for a new species. MIF serological typing is the standard taxonomic test used for identification of rickettsial species (16), and on the basis of our results, as well as previous results (4), there is justification to consider the Swiss agent a new species. The results of the SDS-PAGE protein analysis, Western blot assays performed with specific polyclonal mouse sera, and polymerase chain reactionrestriction fragment length polymorphism analysis provide additional support.

Description of Rickettsia helvetica sp. nov. Rickettsia helvetica (hel.ve'ti.ca. N.L. adj. helvetica, pertaining to Helvetia, the Latin name of Switzerland, where the I. ricinus ticks providing the first isolates were collected) is an obligately intracellular bacterium which grows in several organs of the tick I. ricinus and in Vero, L929, and chicken embryo fibroblasts cells, as well as in yolk sacs. It can be cultured in Earle's minimum essential medium supplemented with $4 \%$ fetal calf serum and $2 \mathrm{mM}$ L-glutamine at $32^{\circ} \mathrm{C}$. Infected cells stained by the Gimenez method are diplococci or rod shaped (4). As determined by electron microscopy $(4,8), R$. helve-

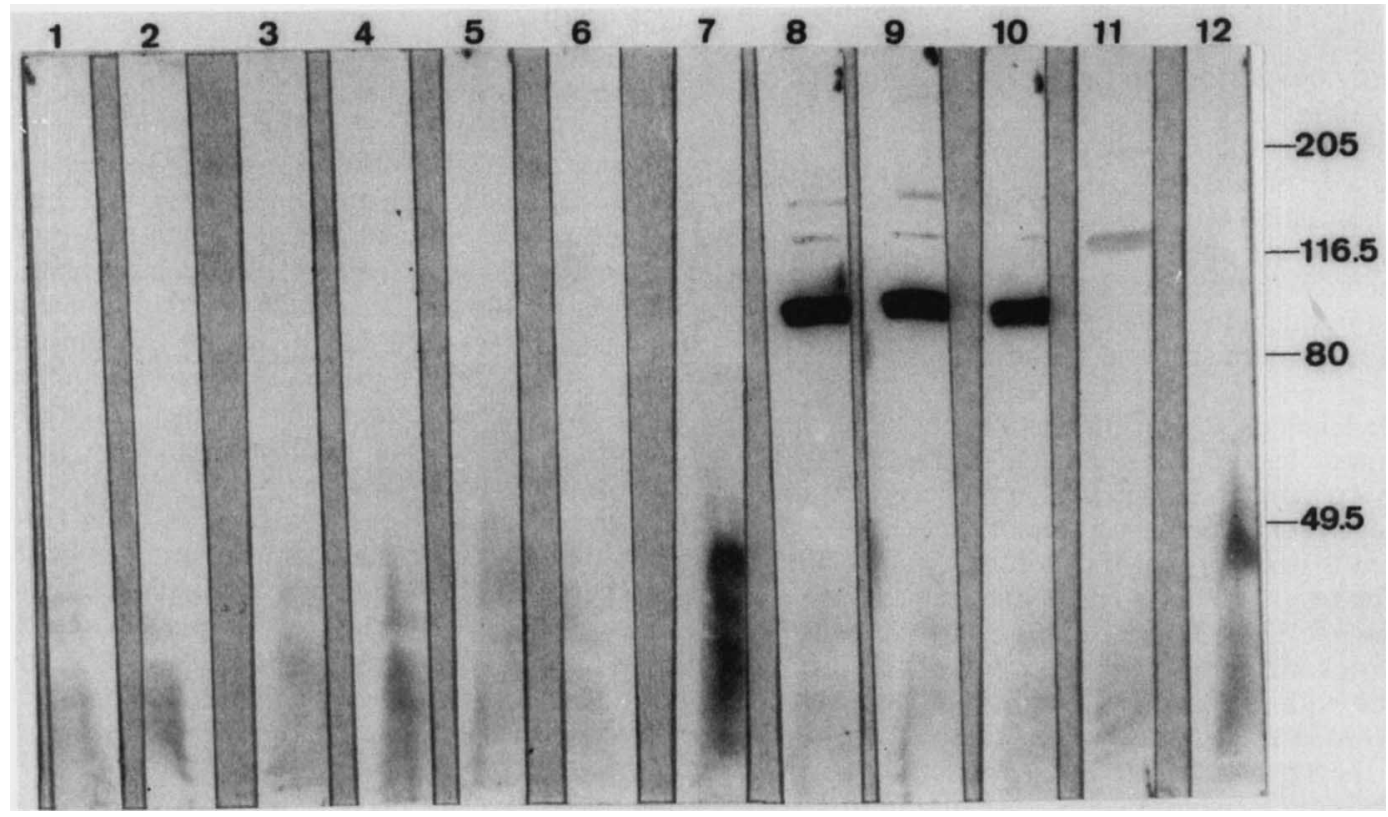

FIG. 2. Western blot of pooled mouse antisera to $R$. helvetica (Swiss agent) strain $\mathrm{C}^{\mathrm{T}}$ against $R$. conorii (lane 1 ), $R$. sibirica (lane 2), $R$. slovaca (lane 3), R. montana (lane 4), R. rhipicephali (lane 5), $R$. australis (lane 6), $R$. akari (lane 7), $R$. helvetica (Swiss agent), strain C3 ${ }^{\mathrm{T}}$ (lane 8), isolate Ix 10 (lane 9), isolate Ix 12 (lane 10), $R$. japonica (lane 11), and " $R$. massiliae" (lane 12). Molecular masses (in kilodaltons) are indicated on the right. 


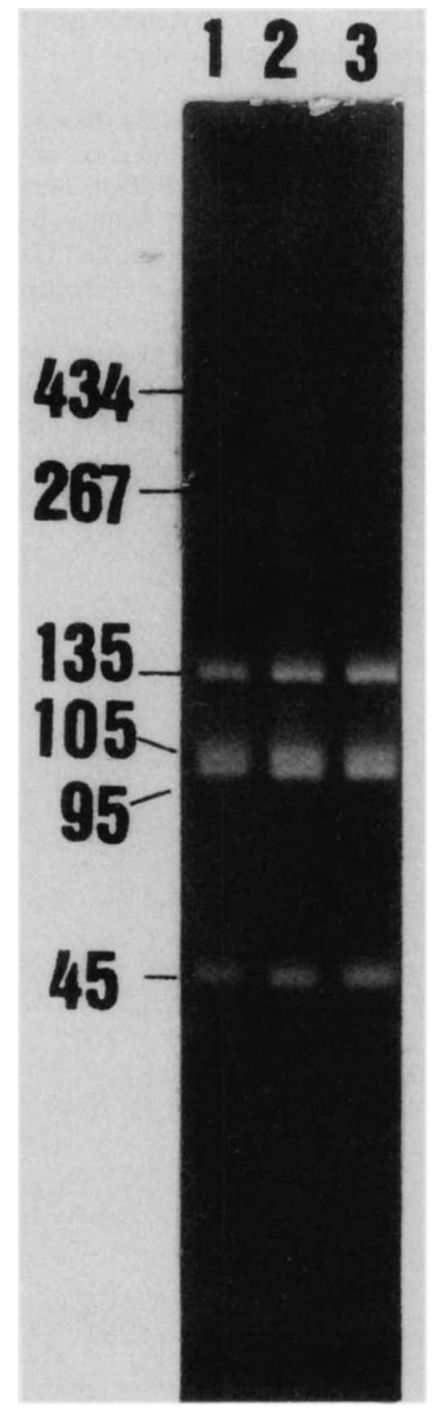

FIG. 3. Ethidium bromide-stained polyacrylamide gels of AluI digests of rickettsial DNAs amplified by using the Rp. CS.877pCS.1258n primer pair. $R$. helvetica $\mathrm{C}^{\mathrm{T}}$ (lane 1), tick isolate $\mathrm{Ix} 10$ (lane 2), and tick isolate Ix 12 (lane 3) produced profiles typical of the SFG rickettsiae, with four bands at 45, 95, 105, and 135 bp. Molecular sizes (in base pairs) are indicated on the left.

tica is free in the cytoplasm and has a trilaminar cell wall, a microcapsular layer, a prominent plasma membrane, and occasionally a slime layer. The cytoplasm contains ribosomes, and the nuclear material may appear to be reticulated. The presence of the organism can be demonstrated by an indirect MIF assay performed with polyclonal mouse sera. Mouse antisera against $R$. helvetica show little or no (4) cross-reactivity with other SFG rickettsiae. The SDS-PAGE proteins profiles are distinct from those of other SFG rickettsiae, and Western blots also exhibit unique profiles. The organism is pathogenic for some rodents, including Microtus pennsylvanicus, Microtus arvalis, and Apodemus flavicollis (12). No information is available about the possible pathogenicity of this organism for other animals or humans. Although high seroprevalence (9.5 to $32.5 \%)$ of antibodies to SFG rickettsiae has been observed among wild and domestic animals in Switzerland, there is no information about possible mammalian reservoirs (13).
The type strain is strain C3 (Reference Center for Rickettsioses, Marseille, France). It is one of the first strains of this species isolated at the Rocky Mountain Laboratories from $I$. ricinus ticks collected in the Staatswald, Bern, Switzerland.

\section{ACKNOWLEDGMENTS}

We thank the Institut de Zoologie, Neuchâtel, Switzerland, which provided the ticks. This study was supported by the Fonds National Suisse de la Recherche Scientifique and the Société Académique Vaudoise.

\section{REFERENCES}

1. Anacker, R. L., R. N. Philip, E. Casper, W. J. Todd, R. E. Mann, M. R. Johnston, and C. J. Nauck. 1983. Biological properties of rabbit antibodies to a surface antigen of Rickettsia rickettsii. Infect. Immun. 40:292-298.

2. Beati, L., M. Eremeeva, X. J. Yu, H. Tissot Dupont, and D. Raoult. 1992. Rickettsia massiliae (sp. nov.). Abstr. 10th Sesquiannu. Meet. Am. Soc. Rickettsiol. Rickettsial Dis.

3. Beati, L., J.-P. Finidori, B. Gilot, and D. Raoult. 1992. Comparison of microimmunofluorescence serologic typing, sodium dodecyl sulfate-polyacrylamide gel electrophoresis, and polymerase chain reaction followed by restriction fragment length polymorphism analysis for identification of rickettsiae: characterization of two new rickettsial strains. J. Clin. Microbiol. 30:1922-1930.

4. Burgdorfer, W., A. Aeschlimann, O. Péter, S. F. Hayes, and R. N. Philip. 1979. Lxodes ricinus vector of a hitherto undescribed spotted fever group agent in Switzerland. Acta Trop. 36:357-367.

5. Fiset, P., R. A. Ormsbee, R. Silbermann, M. Peacock, and S. H. Spielman. 1969. A microagglutination technique for detection and measurement of rickettsial antibodies. Acta Virol. 13:60.

6. Gimenez, D. F. 1964. Staining rickettsiae in yolk-sac cultures. Stain Technol. 39:135-140.

7. Goldwasser, R. A., Y. Steinman, W. Klingberg, T. A. Swartz, and M. A. Klingberg. 1974. The isolation of strains of rickettsiae of the spotted fever group in Israel and their differentiation from other members of the group by immunofluorescence methods. Scand. J. Infect. Dis. 6:53-62.

8. Hayes, S. F., W. Burgdorfer, and A. Aeschlimann. 1980. Sexual transmission of spotted fever group rickettsiae by infected male ticks: detection of rickettsiae in immature spermatozoa of Lxodes ricinus. Infect. Immun. 27:638-642.

9. Kelly, P. J., L. A. Matthewman, L. Beati, D. Raoult, P. R. Mason, M. Dreary, and R. Makombe. 1992. African tick-bite fever: a new spotted fever group rickettsiosis under an old name. Lancet 340:982-983.

10. Kelly, P. J., D. Raoult, and P. R. Mason. 1991. Isolation of spotted fever group rickettsias from triturated ticks using a modification of the centrifugation-shell vial technique. Trans. $R$. Soc. Trop. Med. Hyg. 85:397-398.

11. Laemmli, U. K. 1970. Cleavage of structural proteins during the assembly of the head of bacteriophage T4. Nature (London) 227:680-685.

12. Péter, O. 1981. Contribution à la connaissance de l'écologie de la "rickettsie suisse" transmise par la tique Lxodes ricinus L. 1758. Ph.D. thesis. Faculty of Sciences, University of Neuchâtel, Neuchâtel, Switzerland.

13. Péter, O. 1985. Présence d'anticorps contre la "rickettsie suisse"' chez les mammifères sauvages et domestiques du Canton de Neuchâtel. Schweiz. Arch. Tierheilkd. 127:461-468.

14. Péter, O., D. Raoult, and B. Gilot. 1990. Isolation by sensitive centrifugation cell culture system of 52 strains of spotted fever group rickettsiae from ticks collected in France. J. Clin. Microbiol. 28:1597-1599.

15. Péter, O., J. C. Williams, and W. Burgdorfer. 1985. Rickettsia helvetica, a new spotted fever group rickettsia: immunochemi$\mathrm{cal}$ analysis of the antigens of 5 spotted fever group rickettsiae, p. 99-105. In J. Kazar (ed.), Rickettsiae and rickettsial diseases. Slovak Academy of Sciences, Bratislava, Czechoslovakia. 
16. Philip, R. N., E. A. Casper, W. Burgdorfer, R. K. Gerloff, L. E. Hughes, and E. J. Bell. 1978. Serologic typing of rickettsiae of the spotted fever group by indirect microimmunofluorescence. J. Immunol. 121:1961-1968.

17. Regnery, R. L., C. L. Spruill, and B. D. Plikaytis. 1991. Genotypic identification of rickettsiae and estimation of intraspecies sequence divergence for portions of two rickettsial genes. J. Bacteriol. 173:1576-1589.

18. Robertson, R. G., and C. L. Wissemann. 1973. Tick-borne rickettsiae of the spotted fever group in West Pakistan. Am. J. Epidemiol. 97:55-64.

19. Teysseire, N., C. Chiche-Portiche, and D. Raoult. 1992. Intracellular movements of Rickettsia conorii and Rickettsia typhi based on actin polymerization. Res. Microbiol. 143:821-829.

20. Towbin, H., T. Staehelin, and J. Gordo. 1979. Electrophoretic transfer of proteins from polyacrylamide gels to nitrocellulose sheets: procedure and some applications. Proc. Natl. Acad. Sci. USA 76:4350-4354.

21. Webb, L., M. Carl, D. M. Malloy, G. A. Dasch, and A. F. Azad. 1990. Detection of murine typhus infection in fleas using polymerase chain reaction. J. Clin. Microbiol. 28:530-534.

22. Weiss, E., J. C. Coolbaugh, and J. C. Williams. 1975. Separation of viable Rickettsia typhi from yolk sac and L-cell host components by Renografin density gradient centrifugation. Appl. Microbiol. 30:456-463.

23. Yu, X., D. H. Walker, and T. R. Jerrells. 1990. Polypeptides constituting the antigenic basis for identification of Rickettsia sibirica species by the standard serotyping method for spotted fever group rickettsiae. Acta Virol. 34:71-79. 